\title{
The accuracy of parameters determined with the core-sampling method: Application to Voronoi tessellations
}

\author{
A.G. Doroshkevich ${ }^{1}$, S. Gottlöber ${ }^{2}$, and S. Madsen ${ }^{3}$ \\ 1 Theoretical Astrophysics Center, Juliane Maries Vej 30, DK-2100 Copenhagen Ø, Denmark \\ 2 Astrophysikalisches Institut Potsdam, An der Sternwarte 16, D-14482 Potsdam, Germany \\ 3 Copenhagen University, Astronomical Observatory, Juliane Maries Vej 30, DK-2100 Copenhagen $\varnothing$, Denmark
}

Received May 14; accepted October 10, 1996

\begin{abstract}
The large-scale matter distribution represents a complex network of structure elements such as voids, clusters, filaments, and sheets. This network is spanned by a point distribution. The global properties of the point process can be measured by different statistical methods, which, however, do not describe directly the structure elements.

The morphology of structure elements is an important property of the point distribution. Here we apply the core-sampling method to various Voronoi tessellations. Using the core-sampling method we identify oneand two-dimensional structure elements (filaments and sheets) in these Voronoi tessellations and reconstruct their mean separation along random straight lines. We compare the results of the core-sampling method with the a priori known structure elements of the Voronoi tessellations under consideration and find good agreement between the expected and found structure parameters, even in the presence of substantial noise. We conclude that the core-sampling method is a potentially powerful tool to investigate the distribution of such structure elements like filaments and walls of galaxies.
\end{abstract}

Key words: large-scale structure of Universe - methods: statistical

\section{Introduction}

The first deep galaxy surveys which have become available have shown that the galaxies are not at all homogeneously distributed on large scales. Large voids, small filaments, massive clusters, and walls of galaxies can be observed. How these structures have evolved from the initially nearly homogeneous universe is one of the central

Send offprint requests to: S. Gottlöber, sgottloeber@aip.de problems in modern cosmology. Many theoretical models of structure formation have been suggested. Powerful mathematical statistical methods are necessary in order to describe this structure, to obtain its quantitative characteristics, to compare the theoretical models and simulations with observational catalogues, and, eventually, to find the correct theory of structure formation.

The standard approach for testing models is to define a point process which can be characterized by statistical methods. This could be the distribution of galaxies of a specific type in deep surveys or clusters of galaxies. In order to compare models of structure formation, the different distribution of dark matter particles in N-body simulations could be tested as well.

The most widely used statistics are the n-point correlation functions, the counts-in-cells method, and the void probability function. The geometrical and topological properties of the point distribution can be investigated by the percolation technique (Zeldovich et al. 1982), the minimal spanning tree (Barrow et al. 1985), the genus of the smoothed density field (Gott et al. 1986, 1989), and the Minkowski functionals (Mecke et al. 1994).

All these different statistics measure the global properties of a point process. However, when looking at a point process we also see different structures. The morphology of these structures is an important property of the point distribution. (Note also the warning of Barrow \& Bhavsar (1987) that we tend to see by eye structures which do not exist). Therefore, it is important to develop statistics which distinguish between different structure elements in a point distribution and find their typical scales (if any exists).

In this direction a first attempt was made by Vishniac (1986). He used the moments of a point distribution in a window to measure the amount of filaments in twodimensional galaxy distributions. Recently, this method was generalized to three-dimensional point distributions (Luo \& Vishniac 1995). Both the number of filaments and walls and typical scales of the structure can be found. 

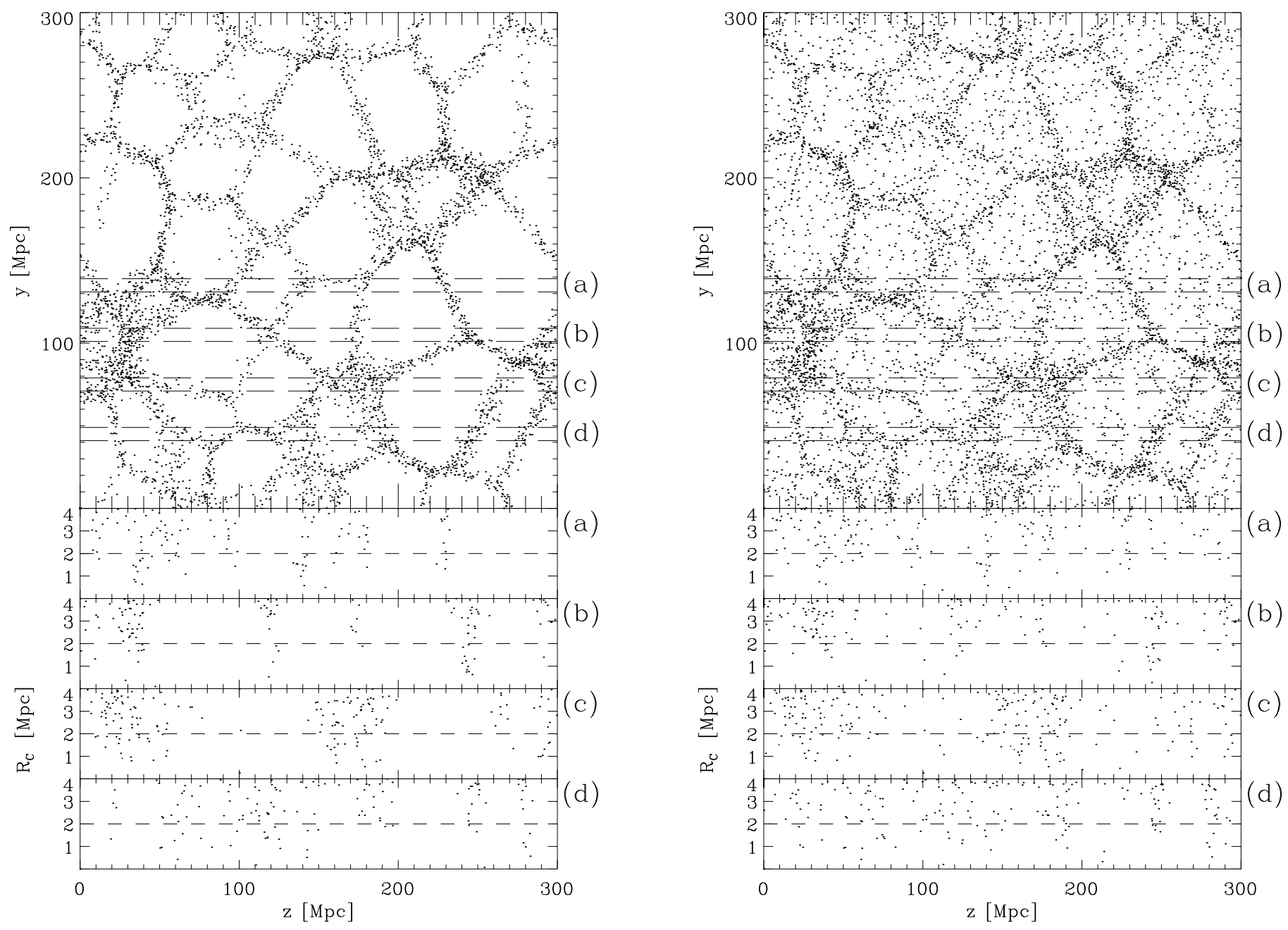

Fig. 1. Top: a slice of $8 \mathrm{Mpc}$ thickness, bottom: radial particle distribution in the four cylinders shown at the top part of the figure (the dashed line indicates the minimum core radius used in the analysis), left: only walls, right: walls with a background of randomly distributed particles

Minkowski functionals are also efficient discriminators for idealized one-, two- or three-dimensional structure elements (Schmalzing et al. 1996). However, using Minkowski functionals it is difficult to extract such structure elements from a superposition of all possible elements including Poisson noise.

Using the core-sampling method (Buryak et al. 1991; Buryak et al. 1994) one can find structure elements and their typical separation. The method was designed to find one-dimensional filaments and two-dimensional walls within observational surveys of galaxies (Buryak et al. 1994; Doroshkevich et al. 1996) and in simulated samples (Doroshkevich et al. 1997). Here we want to apply the method to an idealized model mimicking many features of the observed patterns in the galaxy distribution. The model employed here is based on the concept of a Voronoi tessellation (Goldwirth et al. 1995). Such a test allows us to compare the input and output structure parameters and, thus, to test how powerfully this method can discriminate structure elements, determine their distribution and typical parameters.

The basic idea of the core-sampling method is to reduce the analysis of a three-dimensional galaxy distribution to the investigation of the distribution of structure elements along random straight lines (the "core"). Such an approach allows us to avoid any discussions and descriptions of the very complicated multiconnected structure as a whole as well as any conventional definition of a "structure element" and "void" in the three-dimensional space. The core-sampling method has to deal only with a one-dimensional point distribution. It allows us to define two typical populations of structure elements, namely, filaments and sheets, and provides us with two objective characteristics of the spatial distribution of these elements, namely the surface density of filaments and the linear density of sheets. The surface density of filaments $\sigma_{\mathrm{f}}$ is simply the mean number of filaments intersecting a unit area of arbitrary orientation, while the linear density of sheets 

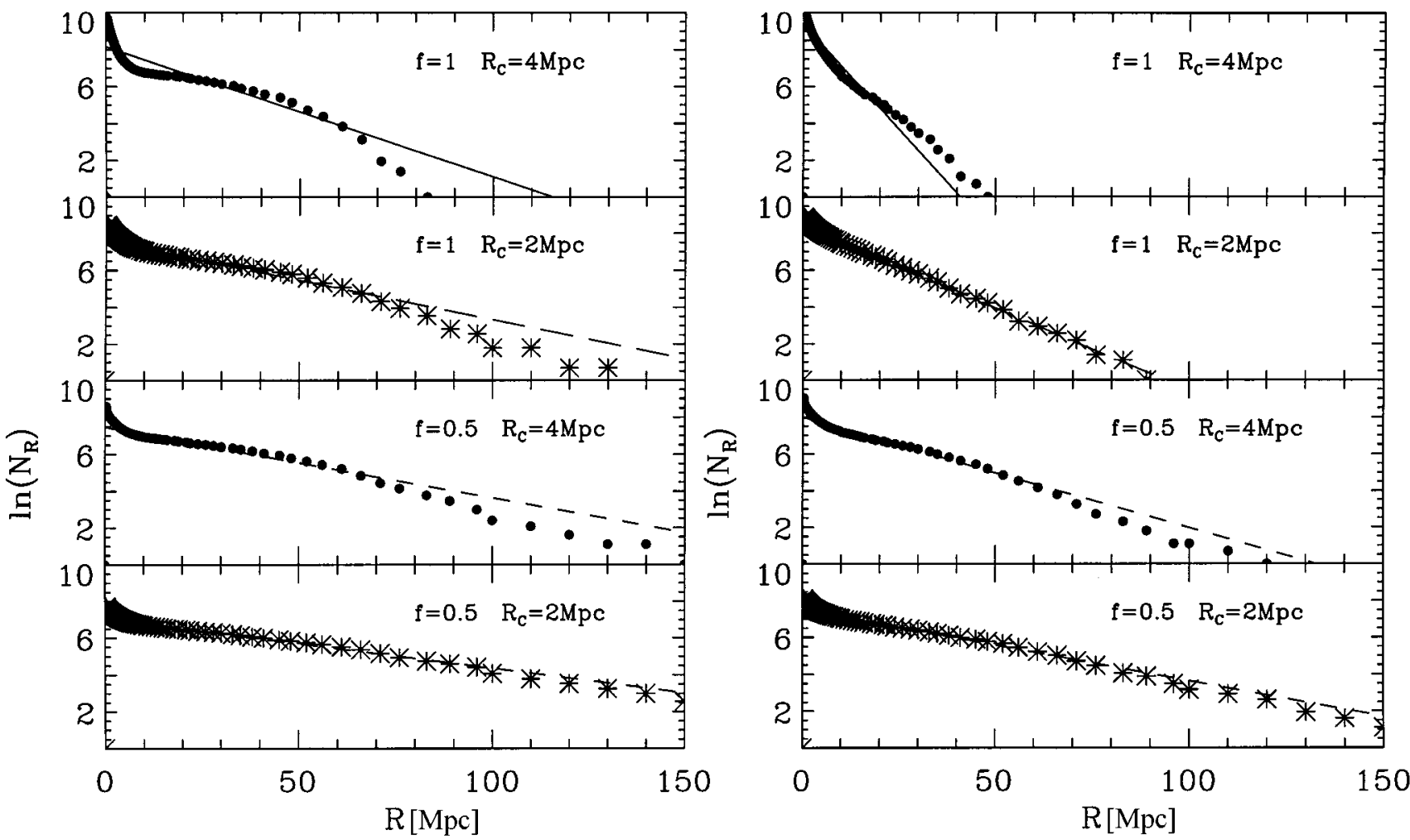

Fig. 2. A fit to the equation $\ln \left(N_{R}\right)=\ln \left(N_{0}\right)-R / R_{0}$ (left: only walls, right: with a background)
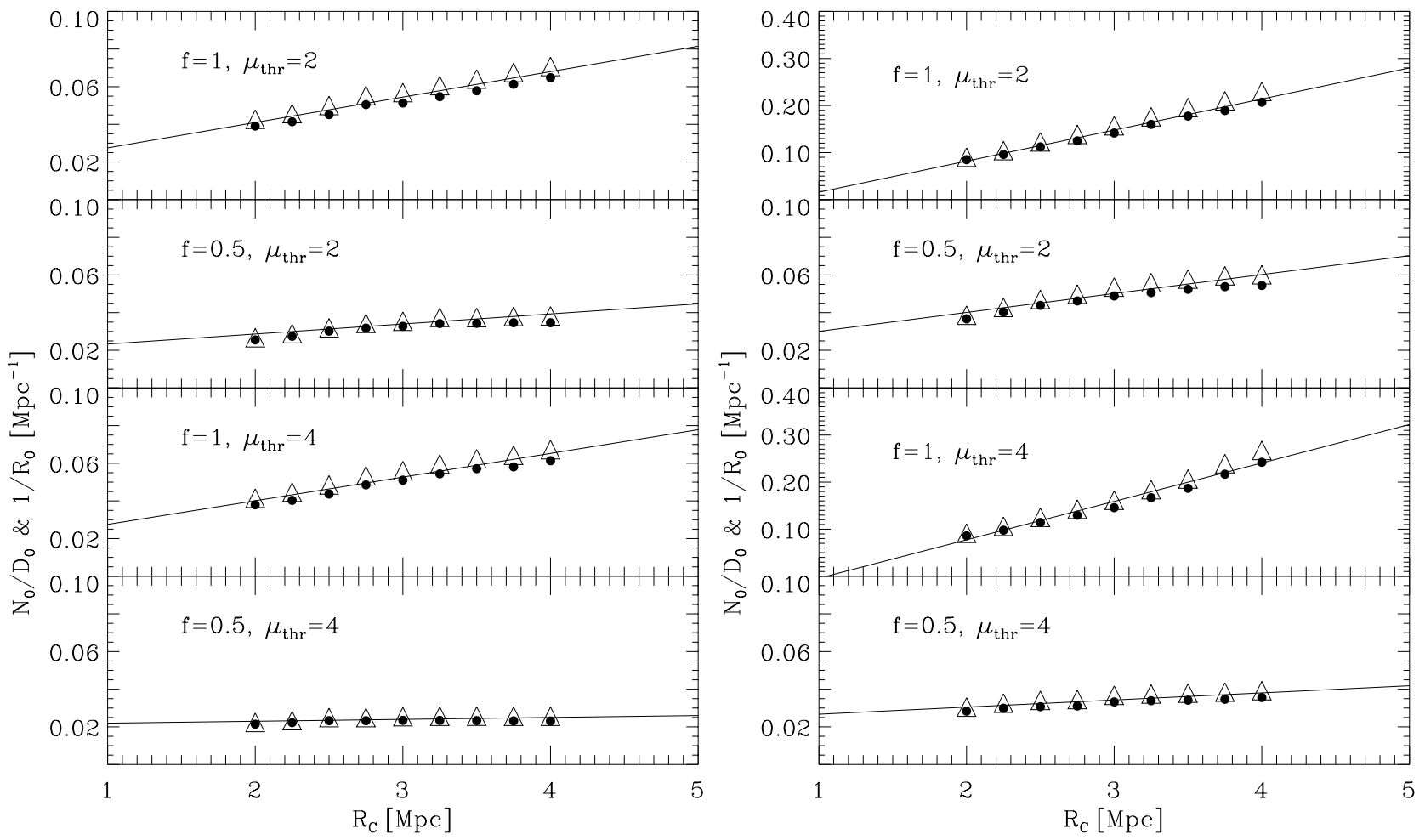

Fig. 3. A fit to the Eqs. (5) and (6) from which $\left.N_{0} / D_{0}\right)$ (dots) and $1 / R_{0}$ (triangles) are obtained, (left: only walls, right: with a background) 

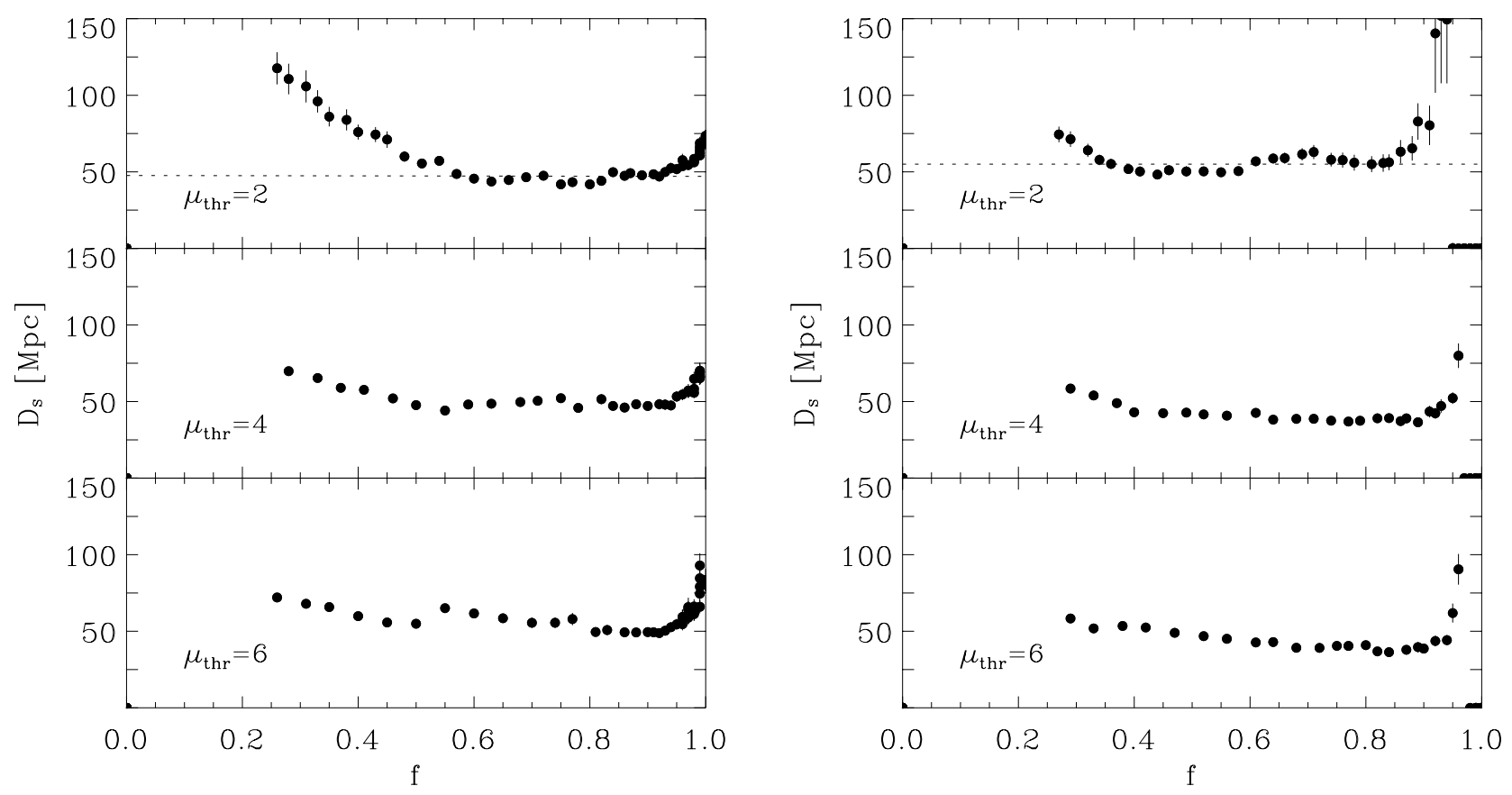

Fig. 4. Mean separation of walls for three multiplicity thresholds (left: only walls, right: with background particles)

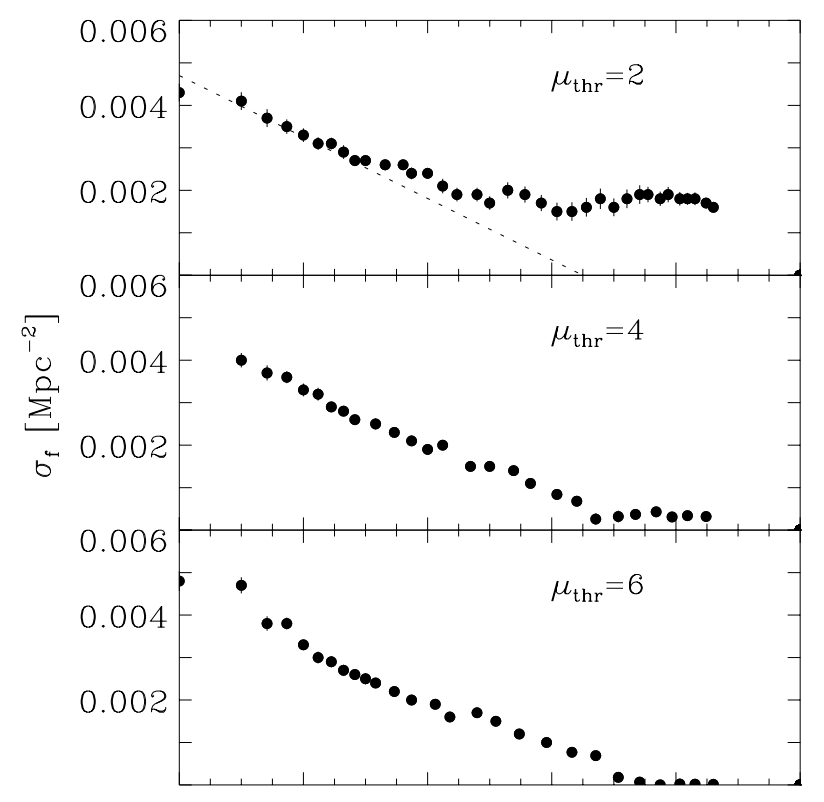

$$
(1 .-\mathrm{f})^{1 / 2}
$$

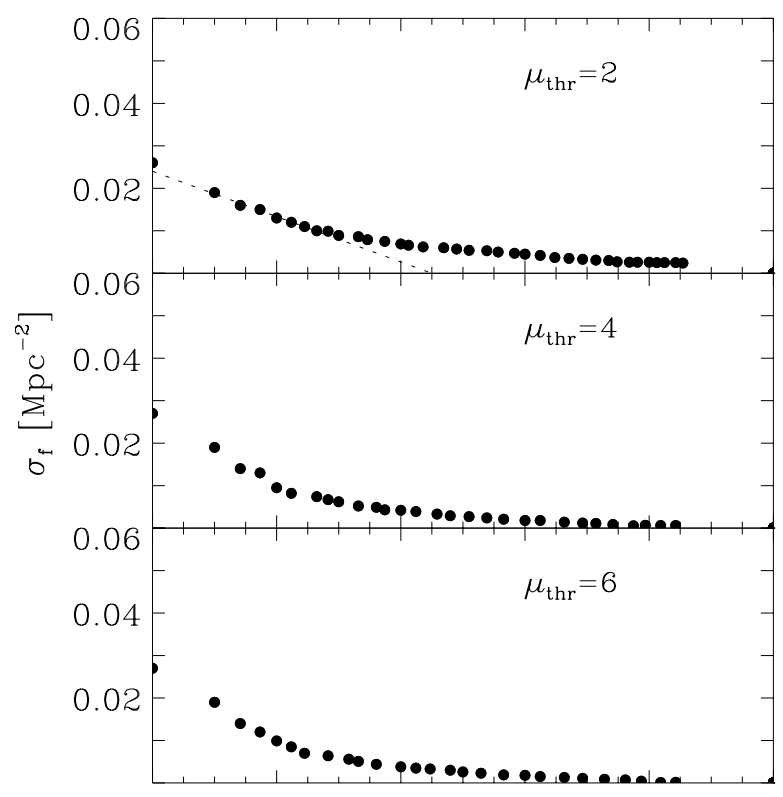

$(1 .-\mathrm{f})^{1 / 2}$

Fig. 5. Mean surface density of apparent filaments for three multiplicity thresholds (left: only walls, right: with background particles) 

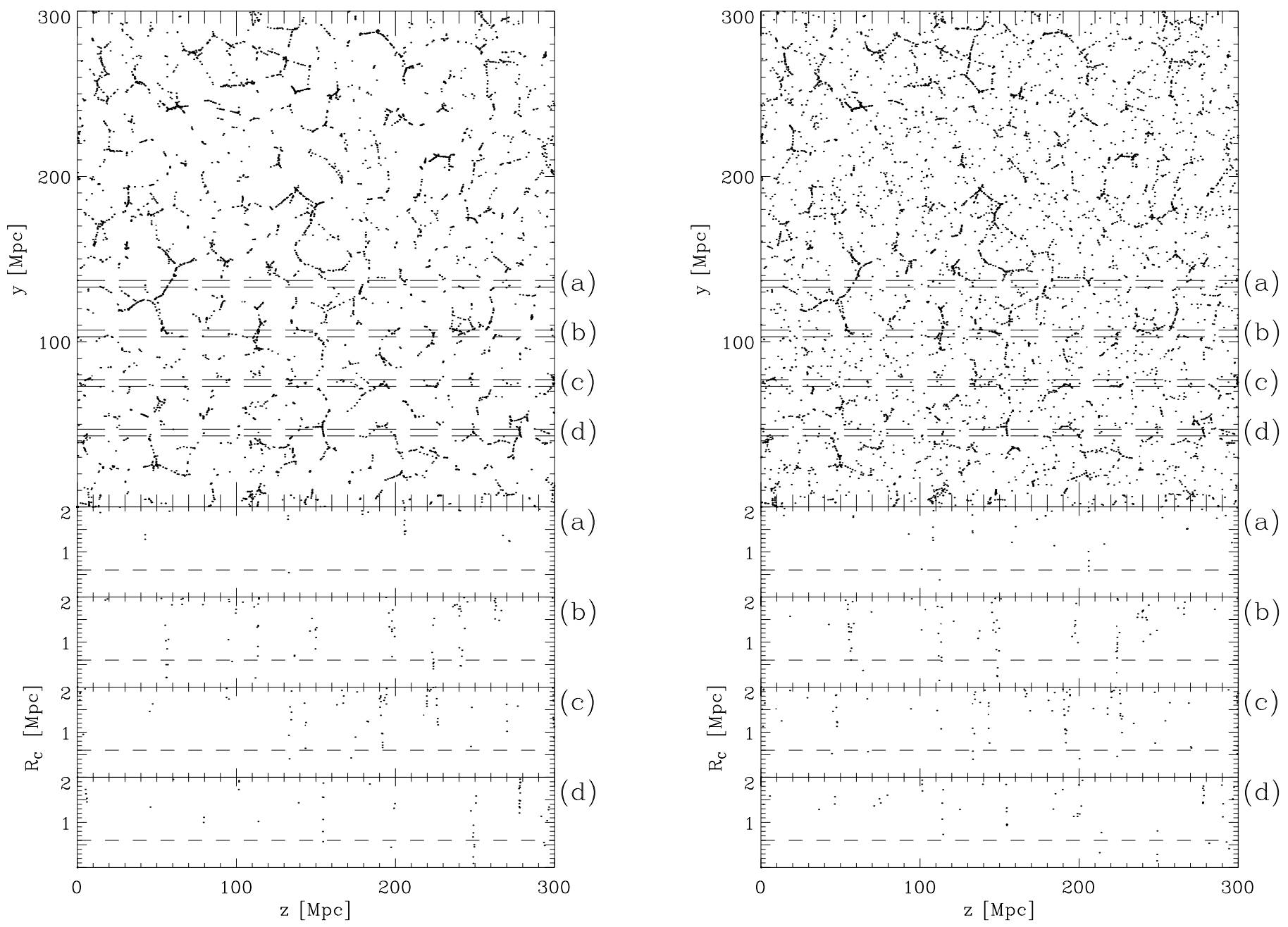

Fig. 6. Top: a slice of $4 \mathrm{Mpc}$ thickness, bottom: radial particle distribution in the four cylinders shown at the top part of the figure (the dashed line indicates the minimum core radius used in the analysis), left: only filaments, right: filaments with a background of randomly distributed particles

$\sigma_{\mathrm{s}}$ is simply the mean number density of sheets crossing an arbitrary straight line. These characteristics provide a local description of a random three-dimensional network structure. In Sect. 2 we will briefly outline the general properties of this method

The three-dimensional Voronoi tessellation consists of a number of Voronoi cells enclosed by the planes bisecting the lines between the nuclei of neighbouring cells (van de Weygaert 1991). The intersections of these planes are lines and points. Therefore, the three-dimensional Voronoi tessellations consists of three structure elements: the walls around the voids, the edges (intersection of walls) and the nodes. Within the three-dimensional Voronoi tessellation particles may be distributed on each of these structure elements or distributed around them according to a given distribution function. Moreover, the Voronoi tessellation can be superimposed by randomly distributed particles. Thus it is a unique tool for testing algorithms purporting to describe the structure elements of a point distribution.
In Sect. 3 we will briefly describe Voronoi tessellations and the creation of synthetic samples. We apply the coresampling method to these synthetic samples and compare the results with the input structure element. We discuss the accordance between the expected and found structure parameters and conclude that the core-sampling method is a powerful tool to investigate the distribution of structure elements.

\section{The core-sampling method}

\subsection{Basis of core-sampling method}

The mathematical basis for this method had been described by Buryak et al. (1991) and Buryak et al. (1994). A recent detailed discussion can be found in Doroshkevich et al. (1996).

First let us give the salient points of the core-sampling method. 

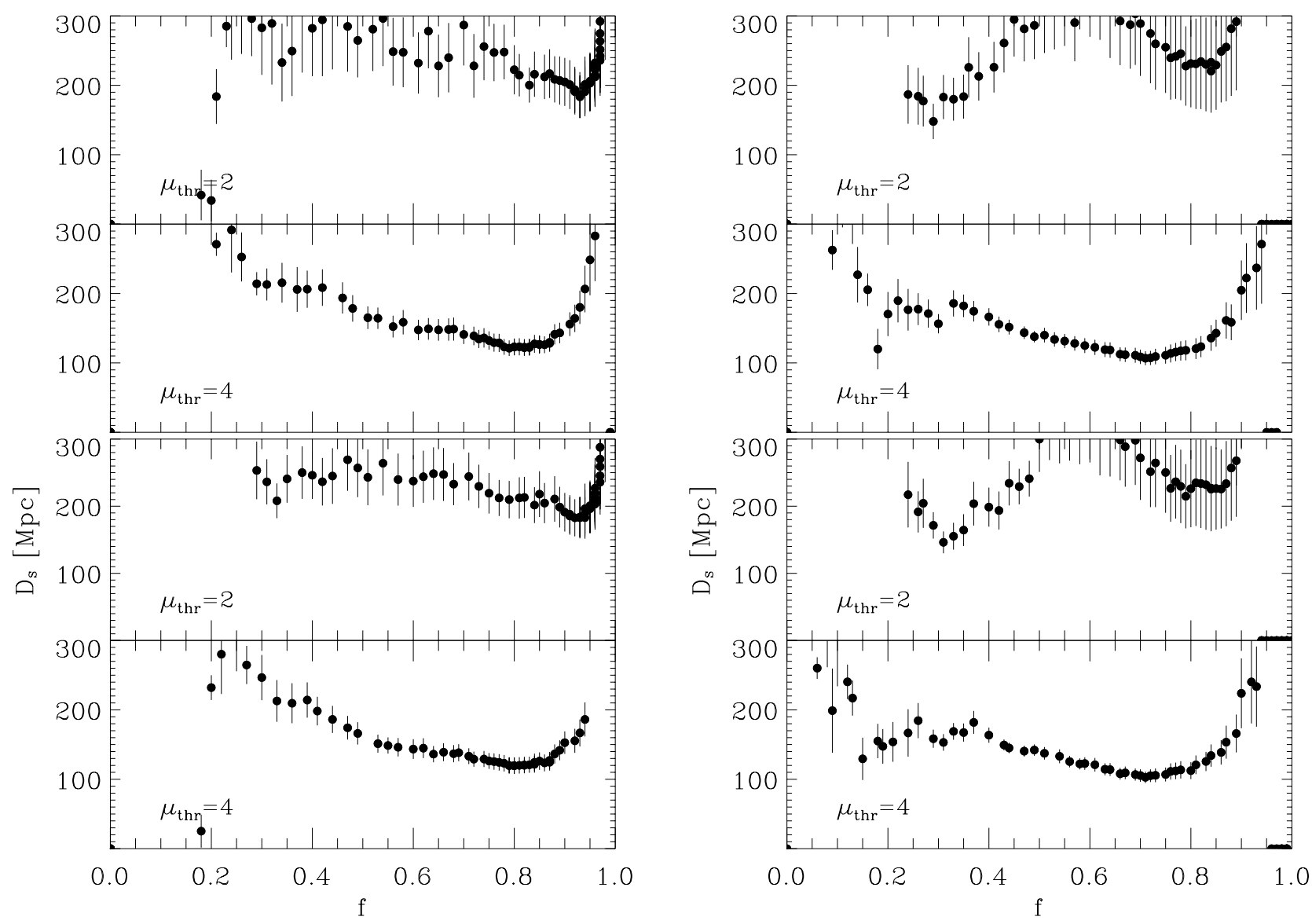

Fig. 7. Mean separation of apparent walls for two multiplicity thresholds (top: radius of filaments 0.2 Mpc, bottom: radius of filaments $0.1 \mathrm{Mpc}$, left: only filaments, right: with background particles)

1. The distribution of structure elements along a random straight line is assumed to be Poissonian. Thus, a 1D cluster analysis is utilized to discriminate the structure elements among the sample of points and to find the number and the mean separation of structure elements.

2. For the following cluster analysis the fields are then all organized into an "equivalent single field" by combining the separate 1D distributions one after the other along a line, with the first point of a field placed on top of the last point of the preceding field.

3 . The dependence of the number and mean separation of structure elements on the diameter of the core allows a rough discrimination between the filament and sheet-like populations of the structure elements and yields the fundamental characteristics of the structure, namely, the surface density of filaments, $\sigma_{\mathrm{f}}$, and the linear density of sheets, $\sigma_{\mathrm{s}}$.

4. The sample of points under consideration is reduced by rejecting poorer and sparser structure elements. In this manner, the mean characteristics can be found as a function of the threshold richness.
Let us emphasize here that in practice the onedimensional analysis is very convenient in many respects. We are using here and in numerical simulations cylinders around straight lines, but they can be easily replaced by cones for observational surveys. Thus, the coresampling method can be directly used to analyze pencil beam surveys as well as almost two-dimensional samples like the slices of the deep Las Campanas survey or real three-dimensional surveys. Moreover, in case of twoand three-dimensional samples it allows measurement of structure parameters in different directions, for instance, both along the line of sight and the transverse direction. Thus, redshift space distortions can be extracted (Doroshkevich et al. 1996).

The assumption of a functional form for the distribution of structure elements along the core is essential for our method, because it enables transformation of the point distribution into a distribution of structure elements. Once this transformation is accomplished, the appropriateness of the assumed functional form can be tested and the values of the functional parameters can be determined. 

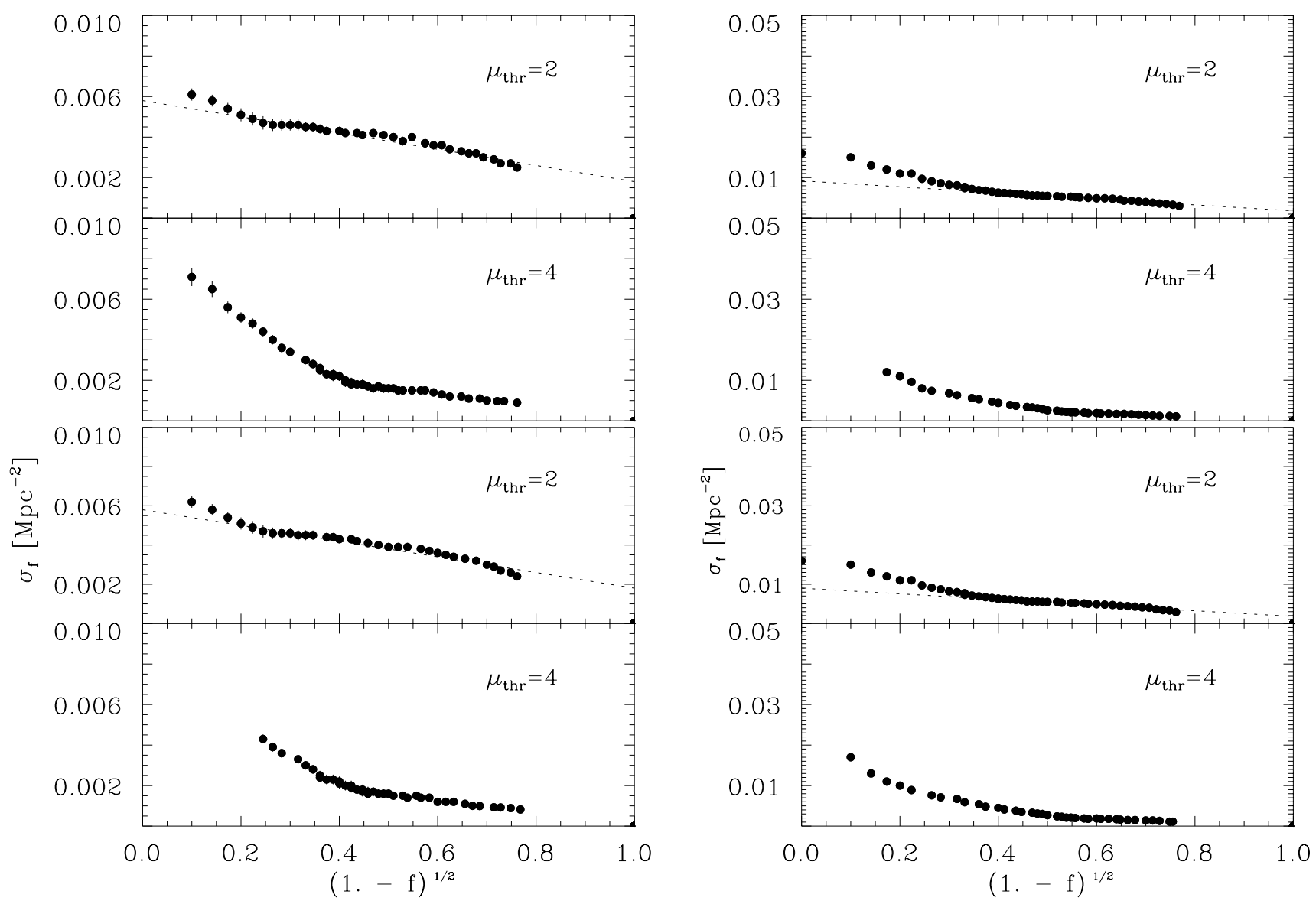

Fig. 8. Mean surface density of filaments for two multiplicity thresholds (top: radius of filaments $0.2 \mathrm{Mpc}$, bottom: radius of filaments $0.1 \mathrm{Mpc}$, left: only filaments, right: with background particles)

As stated above, we assume a 1D Poissonian law for the distribution of structure elements (not galaxies) along the axis of cylinder. It is a simple distribution, and we shall see that it is also a reasonable assumption. Indeed, the Poissonian distribution arises naturally for some theoretical models (White 1979; Buryak et al. 1991) when the mean separation of structure element exceeds the correlation length. The validity of this assumption, however, cannot be tested a priori, and a possible difference between the assumed and the actual distributions depends on the sample in question, thus limiting the precision of the final results. This assumption was valid for all cases in previous investigations. Here we are testing the method with Voronoi tessellations, for which our assumption has to be tested as well.

\subsection{Sample preparation}

The first step of core-sampling analysis is the preparation of a sample with suitable parameters. This sample depends both on the radius of the cylinder $R_{\mathrm{c}}$ and the linking length $l_{\mathrm{c}}$ of the culling procedure. The sample is the basis for the further analysis.
The diameter of the cylinder must be a few times smaller than the expected size of the cells in order to avoid a masking effect due to possible overlapping of projected elements. On the other hand, the cylinder must be wide enough to allow during further analysis the sequential reduction of the radius by a factor of at least 2 , preferably more, because the radius is that diagnostic parameter which allows one to distinguish between filaments and sheets. The number of particles has to be large enough to guarantee stability and reliability of results even for the smallest radius used for analysis. In practice one observes that this can be achieved if a structure element contains, in the mean, at least 5 , or preferably more, particles.

The final step of the sample preparation is sample reduction, which is performed sequentially during the analysis. Sample reduction provides an additional method of distinguishing between different populations of filaments and sheets according to the mean density or, in other words, according to the multiplicity of the structure elements. It has to be performed before any further steps of analysis. 
To do the sample reduction, a 1D cluster analysis of each field is performed with decreasing linking length $l_{\mathrm{c}}$ of the culling procedure. All clusters with fewer members than a constant threshold multiplicity $\mu_{\text {thr }}$ are rejected. Then the remaining number of particles in the sample $N_{\mathrm{r}}$ depends on the current linking length $l_{\mathrm{c}}$ and the threshold $\mu_{\mathrm{thr}}$. For each linking length $l_{\mathrm{c}}$ a certain fraction $f=N_{\mathrm{r}} / N_{\mathrm{a}}$ of points remains $\left(N_{\mathrm{a}}\right.$ is the total number of particles). It is convenient to use the fraction $f$ together with the threshold multiplicity $\mu_{\mathrm{thr}}$, as parameters characterizing the reduced sample.

This procedure reduces the full sample, rejecting more and more points associated with poorer or sparse clumps. Only the tighter clumps are retained for further analysis. This approach emphasizes the local density within the structure elements. It allows the rejection of the lowdensity haloes from the structure elements and sparse structure elements as a whole.

\subsection{Detection of the mean separation of structure elements}

The next step of analysis is the detection of the mean separation of structure elements. At first, the axes of all cylinders are randomly combined to one line by identifying the last point of one axis with the first of the next. At this line a $1 \mathrm{D}$ cluster analysis with increasing linking length $R$ is performed. Assuming a Poisson distribution, the number of clusters $N_{R}$ as a function of $R$ is given by the relation

$\ln \left(N_{R}\right)=\ln \left(N_{0}\right)-R / R_{0}$.

For a true Poissonian sample, these values are related to the length of the sample, $D_{0}$, defined by the nearest and the farthest points in an average field, via the relationship

$N_{0} / D_{0}=1 / R_{0}$.

Thus, the difference between the linear density of structure elements $N_{0} / D_{0}$ and their mean separation $R_{0}$ obtained from Eqs. (1) and (2) can be considered a measure of the systematic error due to the deviation of the actual from the assumed (Poissonian) distribution of structure elements along the analyzed cylinder. To decrease this error we use an automatic procedure to find the optimal interval for $R$ for the fit to Eq. (1). The upper limit to this interval is fixed by the input of a minimum number $N_{R} \geq N_{\min }$, whereas the lower limit can vary and is defined by the condition

$1-\epsilon \leq R_{0} N_{0} / D_{0} \leq 1+\epsilon$

where $\epsilon$ is a desirable precision of fitting parameters. In general, a reasonable precision $(\approx 10 \%)$ can be achieved in the analysis.

If a desirable precision cannot be achieved, the reason is usually that the distribution used is far from Poissonian and the sample under consideration must be changed (e.g., by changing the cylinder radius).

\subsection{Identification of structure elements}

The final step of analysis is the discrimination of filaments and sheet-like structure elements and determination of the parameters $\sigma_{\mathrm{f}}$ and $\sigma_{\mathrm{s}}$ for both populations. To this end, we use a simple geometrical model for the structure elements (Buryak et al. 1994). According to this model, in each narrow cylinder the structure can be considered as a system of randomly distributed lines (filaments) and planes (sheets) which contain all points. In this model, the filaments are considered straight lines, and the sheets are considered flat planes. Of course, this approach is limited, and it cannot be used as an accurate description of the true matter distribution on large scales; one can best characterize it as an intermediate step between the local description of the matter distribution with density and velocity fields and the global description obtained by the topology or Minkowski techniques.

As it was mentioned above, we characterize the random distribution of straight lines (filaments) by their surface density, $\sigma_{\mathrm{f}}$, i.e., the mean number of lines intersecting an unit area of arbitrary orientation. The random distribution of planes can be characterized by the linear density, $\sigma_{\mathrm{s}}$, i.e., the mean number density of planes (sheets) crossing an arbitrary straight line. Equivalently, we can use the values $D_{\mathrm{s}}=\sigma_{\mathrm{s}}^{-1}$ and $D_{\mathrm{f}}=\sigma_{\mathrm{f}}^{-1 / 2}$ as typical measures of the mean separation of structure elements. To characterize the structure as a whole we can estimate the mean separation of structure elements - filaments and sheets combined - by $\left\langle D_{\mathrm{fs}}\right\rangle$, which can be thought of as the diameter of a sphere containing, on average, two structure elements,

$<D_{\mathrm{fs}}>=\frac{2}{\sigma_{\mathrm{s}}+\sqrt{\sigma_{\mathrm{s}}^{2}+\pi \sigma_{\mathrm{f}}}}$.

The main characteristics of the structure can be obtained by fits to the radial dependence of the linear density of clusters, $N_{0} / D_{0}$, and to the radial dependence of the mean separation of structure elements, $R_{0}$,

$N_{0} / D_{0}=\sigma_{\mathrm{s}}+\pi R_{\mathrm{c}} \sigma_{\mathrm{f}}$

and

$1 / R_{0}=\sigma_{\mathrm{s}}+\pi R_{\mathrm{c}} \sigma_{\mathrm{f}}$

over the range of variation of core radius $R_{\mathrm{c}}$. Here $D_{0}$ is the mean depth of field.

Clearly, these parameters also depend on the sample being studied. In our analysis, Eqs. (5) and (6) were fitted by a maximum likelihood technique, and the resulting mean values of $\sigma_{\mathrm{f}}$ and $\sigma_{\mathrm{s}}$ were accepted as the final estimates of the structure parameters. The difference of the estimates from Eqs. (5) and (6) was included into the errors of the final values. 


\subsection{Methodological remarks}

For the idealized model considered above, the coresampling method would be expected to reproduce well the characteristics of the sample under consideration. However, in reality, several factors may distort the final results. For example, filaments may be apparently detected as sheets, if they pass through the center of the core. Also the noise of randomly distributed particles must be taken into account. Therefore, a specific technique must be used to obtain stable results.

The analysis of the Las Campanas Redshift Survey (Doroshkevich et al. 1996) has shown that the stability of core-sampling with respect to various accidental or systematic variations of density is very high. During the sequential random rejection of particles, the final estimates of mean separation of structure elements increased only as $f^{-1 / 3}$. In general, the precision and stability of the core-sampling method depends on the density contrast in structure elements relative to the mean number density of points. In case of pure Poissonian point distribution the "structure" parameters have been found with errors of about 50\% (Buryak et al. 1994). Similar errors were found for the Soneira-Peebles model. On the contrary, for the observed Las Campanas Redshift Survey errors were found to be about $10 \%$ only.

1. Sample preparation.

During the first step of analysis, when the sample is prepared, one has to check whether the resulting $1 \mathrm{D}$ cluster distribution is Poissonian as assumed. One has to ensure this requirement by the variation of the radius of cylinder and the range of fit in Eq. (1). During the further analysis the range of core radii used for the fit of Eqs. (5) and (6) is important to get the two values in agreement. These questions have to be solved before the final analysis. This means that during each of the intermediate steps leading to Figs. 1 to 3, the validity of all assumptions must be checked. Otherwise, the final result will be meaningless.

2. The surface density of filaments.

The richness of a filament in the core depends both on the actual properties of the point distribution analyzed and the geometry, i.e. the position and orientation of the filament relative to the core. Only the first piece of information is of interest; the geometry can be characterized analytically and used to improve the stability of the method.

The surface density of filaments $\sigma_{\mathrm{f}}\left(f, \mu_{\mathrm{thr}}\right)$ depends on the fraction of particles retained in the sample and the multiplicity threshold. The main characteristic of filamentary structure is the full surface density of filaments, $\sigma_{\mathrm{f}}(1,1)$. However, at this point the noise is usually high and it is desirable to improve the estimate of this value.

Basing on the geometrical model described above one can calculate the distribution function for the length of intersection of identical filaments with the core. Thus, calculating the dependence of both the fraction $f$ and the surface density $\sigma_{\mathrm{f}}$ on this length, we find in linear approximation

$\sigma_{\mathrm{f}}(f) \approx \sigma_{\mathrm{f}}(1)\left(1-\sqrt{\frac{2}{3}}(1-f)^{1 / 2}+\ldots\right)$.

This relation estimates the fraction of poor filaments for the ideal case without noise. The linear fit of Eq. (7) over some range of $f$ allows one also to improve the estimate of $\sigma_{\mathrm{f}}(1)$ for a moderate noisy sample. However, in the case of very noise samples with a significant fraction of filaments consisting only of one point, it can be only used for small linking lengths, $l_{\mathrm{c}}$, of the culling procedure. Indeed, if $l_{\mathrm{c}} \leq R_{0}$ the main part of noise particles will be rejected (see Fig. 8, right). Evidently, for smaller values of $f$, the function $\sigma_{\mathrm{f}}(f)$ resembles the actual properties of the filaments (see, e.g. Doroshkevich et al. 1996).

3. The linear density of sheets.

The richness of the elements of the sheet-like population both in observational data and simulations is usually quite high. Thus, the linear density of sheets can be estimated for a wide range of parameters $f$ and $\mu_{\text {thr }}$, providing more reliable results. However, even in this case the final results are sometimes distorted because during reduction the fields are shortened as a result of the appearance of empty edges. To avoid these distortions, a correction procedure (see Doroshkevich et al. (1996) was used.

The next problem is a filament population masquerading as sheets. These apparent sheets are detected by the program if filaments cross the cylinder near its axes. Obviously, this effect will be proportional to the thickness of the filaments. The surface density $\sigma_{\mathrm{s}}^{\mathrm{a}}$ of these apparent sheets is $\sigma_{\mathrm{s}}^{\mathrm{a}}=\pi \sigma_{\mathrm{f}} r_{\mathrm{f}}$, where $r_{\mathrm{f}}$ is the radius of the filament (Buryak et al. 1994). Usually the influence of this effect is negligible, but it can become important for thick filaments. Moreover, apparent sheets can be generated by the branch points (knots) of filamentary structure or other clumps in the point distribution if their size is comparable to the diameter of the core. The contribution of these knots can be estimated as $\sigma_{\mathrm{s}}^{\mathrm{a}}=\pi n_{\mathrm{clm}} r_{\mathrm{clm}}^{2}$, where $n_{\mathrm{clm}}$ and $r_{\text {clm }}$ are respectively the volume density and the effective radius of the clump. For a random network structure, the volume density of the knots is approximately $n_{\mathrm{clm}} \approx \sigma_{\mathrm{f}}^{3 / 2}$.

\section{Analysis of Voronoi-tessellations}

\subsection{Construction of Voronoi tessellations}

We have used the code developed by R. Van de Weygaert (1991, 1994, see also Goldwirth et al. 1995) in order to construct Voronoi tessellations. Although the code has been developed to allow for much more sophisticated cases, we 
limit ourselves to the simplest case where a given number of points is distributed in walls or filaments of a given thickness. To mimic noise we have added an Poisson distribution of particles in the whole box.

Using the same nuclei of the Voronoi tessellation, the filaments (defined as the edges of the walls) would have approximately the same separation as the walls (contrary to what we expect in nature). Therefore, we did not combine a filamentary structure with a wall-like structure in one realization, but made a second realization for filaments alone. Note also that the filaments of a Voronoi tessellation are not distributed like filaments of real galaxies. Nevertheless the points of these Voronoi tessellation form one-dimensional structure elements with a priori known properties which should be recovered by the core sampling method.

The mean diameter of the Voronoi cells and the size of the box are free input parameters of the code. For the core-sampling analysis it is only important that the mean number of structure elements along a random line crossing the box be large enough. We have chosen 6 cells and 20 cells for the analysis of walls and filaments, respectively. The analysis itself can be performed in units of the box length. For the graphical representation we have chosen formally a box size of $300 \mathrm{Mpc}$. We have chosen 200000 and 400000 points to represent the walls and the filaments, respectively. Due to the formal definition of the box size these numbers are not directly comparable with the number of galaxies in the corresponding volume. However, in that case the mean number density of points is of the order $10^{-2} \mathrm{Mpc}^{-3}$, i.e of the same order as the galaxy density. Also the the mean separation of structure elements is of the same order as found for galaxies, so that we can indeed draw conclusions from our test to the situation found in observational surveys. The noise level chosen $(50 \%$ for walls and $25 \%$ for filaments) is in these realisations an approximate upper limit for which the known properties can be recovered with high accuracy.

\subsection{Wall distribution}

For the first test, we chose a mean size of the cells of $50 \mathrm{Mpc}$. Our first realization consists of $6^{3}$ randomly distributed cells in box of $300 \mathrm{Mpc}$ length. 200000 points are distributed only in walls around the cells. We chose a Gaussian density profile of the walls with a thickness of $2 \mathrm{Mpc}$. On the left hand side of Fig. 1, a slice of $8 \mathrm{Mpc}$ thickness of this realization is shown. In order to test the stability of the core-sampling method, we made a second realization with 100000 additional points which were randomly distributed (Fig. 1, right).

As explained in Sect. 2.1, the starting point of the core-sampling analysis is the construction of randomly distributed cylinders in the simulation. For illustration, we put four of these cylinders into each of the slices shown in Fig. 1. The diameter of the cylinders is equal to the thickness of the slice $(8 \mathrm{Mpc})$. In the lower part of the figures, the distribution of points in these cylinders is plotted vs. the radius of the cylinder. This radius will be used in the following as one diagnostic parameter as described in Sect. 2. Indeed, for sheet-like structures like the walls in our Voronoi tessellations, the mean number of clusters in the 1D cluster analysis is independent of the core radius. One can clearly see from Fig. 1 that in both cases almost all walls will be identified as walls.

Next, all particles $N_{\mathrm{a}}$ in a given cylinder of radius $R_{\mathrm{c}}$ are projected onto the axis of the cylinder. From Fig. 1 it is clear that there is also noise besides real structures in the resulting one-dimensional point distribution. The degree of noise depends on the chosen parameters of the generated sheets and on the parameters of the core. The noise particles in underdense regions can be removed using the reduction procedure described in Sect. 2.1. The reduced sample at which the further analysis will be performed is characterized by three parameters: the fraction $f$, the threshold $\mu_{\mathrm{thr}}$, and the core radius $R_{\mathrm{c}}$.

Further, we use the iterative fitting procedure described in Sect. 2.2 to find the best fit to Eq. (1). According to our assumption, the resulting clusters must be Poisson distributed for the some range of linking length. However, this assumption must be tested for the samples under consideration. As an example we show in Fig. 2 the fit to Eq. (1) for four sets of parameters $(f=1,0.5, r=4 \mathrm{Mpc}$, $2 \mathrm{Mpc}$ and $\mu_{\mathrm{thr}}=2$ : left for walls and right for walls with randomly distributed points).

Using Eqs. (5) and (6), where $R_{\mathrm{c}}$ is the diagnostic parameter, we determine now the surface density of filaments and the linear density of walls for each pair of parameters $f$ and $\mu_{\mathrm{thr}}$. This procedure is illustrated by Fig. 3. for four pairs of parameters. (Note that each curve of Fig. 2 corresponds to one point in Fig. 3). From Fig. 3 we extract the linear density of walls as the zero point of the curve and the surface density of filaments as the gradient. The fits to Eqs. (5) and (6) were performed over 9 equally spaced values of $R_{\mathrm{c}}$ in the interval $2 \mathrm{Mpc} \leq R_{\mathrm{c}} \leq 4 \mathrm{Mpc}$.

As the final result of the analysis, we present in Fig. 4 the mean distance of the walls $D_{\mathrm{s}}=\sigma_{\mathrm{s}}^{-1}$ and in Fig. 5 the surface density of filaments $\sigma_{f}$ depending on the threshold $\mu_{\text {thr }}$. (Note, that each curve of Fig. 3 provides one point in Fig. 4 and one point in Fig. 5.) The final values of $\sigma_{\mathrm{s}}$ and $\sigma_{\mathrm{f}}$ are listed in Table 1.

Table 1. Test of the wall distribution

\begin{tabular}{ccccc}
\hline$\mu_{\mathrm{thr}}$ & $\begin{array}{c}\sigma_{\mathrm{s}} \\
10^{-2} \mathrm{Mpc}^{-1}\end{array}$ & $\begin{array}{c}\sigma_{\mathrm{f}} \\
10^{-2} \mathrm{Mpc}^{-2}\end{array}$ & $\begin{array}{c}\sigma_{\mathrm{s}}^{\text {noise }} \\
10^{-2} \mathrm{Mpc}^{-1}\end{array}$ & $\begin{array}{c}\sigma_{\mathrm{f}}^{\text {noise }} \\
10^{-2} \mathrm{Mpc}^{-2}\end{array}$ \\
\hline 2 & $2.1 \pm 0.29$ & $0.43 \pm 0.21$ & $1.8 \pm 0.20$ & $2.7 \pm 0.16$ \\
4 & $2.0 \pm 0.13$ & $0.43 \pm 0.19$ & $2.5 \pm 0.29$ & $2.7 \pm 0.16$ \\
6 & $1.8 \pm 0.16$ & $0.48 \pm 0.21$ & $2.1 \pm 0.17$ & $2.7 \pm 0.16$ \\
\hline
\end{tabular}


The noise of apparent filaments is an objective characteristic of the sample under consideration. The population of filaments dominates for all thresholds $\mu_{\mathrm{thr}}$ that resemble the small density contrast in the walls, since the number of particles concentrated into sheets exceeds the noise particles only by a factor of two. In this respect our synthetic models differ from the Las Campanas Redshift Survey, where for high threshold multiplicity the filament population had disappeared, and only the richest sheetlike elements survived.

\subsection{Filament distribution}

As a second test we have investigated a pure filamentary structure and filaments superimposed by randomly distributed particles. To this end we have constructed a Voronoi tessellation with 400000 particles which are distributed at the edges of the cells, and a second one with an additional 100000 randomly distributed particles. For this test we chose a mean size of the cells of 15 Mpc. In Fig. 6 we show a slice of $4 \mathrm{Mpc}$ thickness and four cores of radius $2 \mathrm{Mpc}$. Further steps in the analysis are as above. We made two realisations of Voronoi tessellations containing only filamentary structure: In the first realisation we chose a radius of filaments of $0.2 \mathrm{Mpc}$, in the second $0.1 \mathrm{Mpc}$.

For these synthetic models, we omit the figures corresponding to Figs. 2-3, but present only the final results, i.e. the density of walls $\sigma_{\mathrm{s}}(f)$ and filaments $\sigma_{\mathrm{f}}(f)$ vs. $(1-f)^{1 / 2}$ for two thresholds $\mu_{\mathrm{thr}}=2,4$ and the two different radii of filaments. (see Figs. 7-8). The final structure parameters are given in Table 2 .

Let us note that in this case the mean edge separation along a random straight line is not identical to the mean size of the cell but is of the order of the mean length of the filaments. The mean distance of particles along the filaments in our sample is of the order of $1 \mathrm{Mpc}$. Therefore, we expect that the set of particles forms a broken network structure which can be characterized by the surface density of filaments $\sigma_{\mathrm{f}}(f)$. With a higher mean density we could create also a complete network structure which, however, in reality does not exist.

Since all filaments were created using the same procedure their parameters can vary only statistically. We can use Eq. (7) to determine $\sigma_{\mathrm{f}}(1)$ as is shown in Fig. 8 (dashed line). In case of $\mu_{\mathrm{thr}}=4$ the points corresponding to a large fraction $f$ do not follow the line predicted by Eq. (7). Rejecting these points, a linear fit would predict a somewhat smaller $\sigma_{\mathrm{f}}$. We expect this result, because we are now measuring only the rare high-density filaments.

The noise strongly increases the full surface density of filaments $\sigma_{f}(1)$ (Fig. 8, right). The rejection of (10$15 \%$ ) of particles depresses the noise impact and leads us back to noiseless data. Since most or all observed galaxies are incorporated into structure elements the noise impact is not expected to be severe in catalogues. Following the rejection procedure we can obtain for all realizations the same result $\sigma_{\mathrm{f}}(1)=0.0054$ which corresponds to $13.6 \mathrm{Mpc}$ mean separation (see Fig. 8).

Figure 6 (bottom) indicates that some of the filaments masquerade as sheets. We have discussed this problem already in Sect. 2.4. In this case the error bars strongly increase as a result of the sparse sample statistics of elements which the core-sampling method wrongly detects as sheets (see Fig. 7).

Table 2. Test of the filament distribution

\begin{tabular}{ccccc}
\hline$\mu_{\mathrm{thr}}$ & $\begin{array}{c}\sigma_{\mathrm{s}} \\
10^{-3} \mathrm{Mpc}^{-1}\end{array}$ & $\begin{array}{c}\sigma_{\mathrm{f}} \\
10^{-2} \mathrm{Mpc}^{-2}\end{array}$ & $\begin{array}{c}\sigma_{\mathrm{s}}^{\text {noise }} \\
10^{-3} \mathrm{Mpc}^{-1}\end{array}$ & $\begin{array}{c}\sigma_{\mathrm{f}}^{\text {noise }} \\
10^{-2} \mathrm{Mpc}^{-2}\end{array}$ \\
\hline \multicolumn{5}{r}{$=0.2 \mathrm{Mpc}$} \\
\hline 2 & $4.4 \pm 0.8$ & $0.57 \pm 0.02$ & $5.3 \pm 1.0$ & $0.92 \pm 0.05$ \\
4 & $7.3 \pm 0.8$ & $0.30 \pm 0.02$ & $6.5 \pm 0.6$ & $0.50 \pm 0.04$ \\
\hline \multicolumn{5}{c}{$r=0.1 \mathrm{Mpc}$} \\
4 & $6.1 \pm 0.8$ & $0.31 \pm 0.01$ & $5.5 \pm 1.0$ & $0.49 \pm 0.04$ \\
\hline
\end{tabular}

\section{Conclusions}

We have constructed a series of Voronoi tessellations with different input parameters. Our realisations consist either only of structure elements or of structure elements and noise. We have chosen a number of points comparable with the situation in observational samples and simulations of large scale structure formation, i.e. our realisations correspond to models with all galaxies within structure elements and models with additional randomly distributed galaxies.

In the first two tessellations, only walls were constructed. Using the core-sampling method, we detected walls with a mean separation of $50 \mathrm{Mpc}$ (see Table 1 and Fig. 2) in these synthetic models. This corresponds exactly to the input parameter (the mean void size) of the Voronoi tesselation. The method provided an accurate reconstruction despite the additional noise which was added to the tessellation (Fig. 4, right). On the other hand, the scales of the spurious filaments in the tessellation (Fig. 5) are sensitive to the addition of noise. The specific dependence found appears to be a property of the particular synthetic model generated.

The next four tessellations are all characterized by a mean cell size of $15 \mathrm{Mpc}$. The cells are surrounded only by filaments. Two tessellations were made with filaments of radius $0.2 \mathrm{Mpc}$, two more with $0.1 \mathrm{Mpc}$. Independent of the radii of filaments, we were able to determine the same mean distance of filaments $(13.6 \mathrm{Mpc})$; however, this estimate was slightly reduced by adding randomly distributed points as background (cp. Fig. 8 , dashed lines). The detected spurious walls have distances of the order of the 
box size and very large errors so that they can be easily ruled out.

Our results demonstrate clearly that the core-sampling method allows one not only to determine the structure elements, but also to measure their characteristic mean separation or density almost independently of the influence of a substantial noise component. From the good agreement between the expected and recovered structure parameters, we conclude that the core-sampling method is a powerful tool for further investigations of observational surveys and the determination of structure elements such as filaments and walls of galaxies. Recently, the core-sampling method has been successfully applied both to numerical simulations (Doroshkevich et al. 1997) and the Las Campanas galaxy sample (Doroshkevich et al. 1996).

Acknowledgements. We would like to thank Rien Van de Weygaert for allowing us to use his code for generating Voronoi tessellations and Ron Kates for valuable discussion of the manuscript. This paper was supported in part by Danmark Grundforskningsfond through its support for the establishment of the Theoretical Astrophysics Center. A.G.D. was partly supported by the INTAS grant 93-0068 and by the Center for Cosmoparticle Physics "Cosmion" in the framework of the project "Cosmoparticle Physics". S.G. wishes to express gratitude for the hospitality of the TAC Copenhagen.

\section{References}

Barrow J.D., Bhavsar S.P., Sonoda D.H., 1985, MNRAS 216, 17

Barrow J.D., Bhavsar S.P., 1987, QJRAS 28, 109

Buryak O.E., Demiański M., Doroshkevich A.G., 1991, ApJ 383,41

Buryak O.E., Doroshkevich A.G., Fong R., 1994, ApJ 434, 24

Doroshkevich A.G., Fong R., Gottlöber S., Mücket J.P., Müller V., 1997, MNRAS 284, 633

Doroshkevich A.G., Tucker D., Oemler Jr. A.A., Kirshner R.P., Lin H. Shectman S.A., Landy S.D., Fong R., 1996, MNRAS 283, 1281

Goldwirth G.S., da Costa L.N., Van de Weygaert R., 1995, MNRAS 275, 1185

Gott J.R., Melott A.L., Dickinson M., 1986, ApJ 306, 341

Gott J.R., Miller J., Thuan T.X., et al., 1989, ApJ 340, 625

Klypin A.A., 1987, Sov. Astr. 31, 8

Luo S, Vishniac E., 1995, ApJS 96, 429

Mecke K.R., Buchert T., Wagner H., 1994, A\&A 288, 697

Schmalzing J., Kerscher M., Buchert T., 1996, Course CXXXII: Dark Matter in the Universe. In: Bonometto S., Primack J., Provenzale A. (eds.) Proceedings of the International School of Physics Enrico Fermi, p. 281

Vishniac E., 1986, in Proc. Inner Space/Outer Space, Kolb E.W., Turner M.S., Olive K., Seckel D., Lindey D. (eds.). Chicago:Chicago Univ. Press, p. 190

Van de Weygaert R., 1991, Ph.D. Thesis, University of Leiden Van de Weygaert R., 1994, A\&A 283, 361

Zeldovich Ya.B., Einasto J., Shandarin S., 1982, Nat 300, 407 